\title{
Phaseless Gauss-Newton Inversion for Microwave Imaging
}

This paper was downloaded from TechRxiv (https://www.techrxiv.org).

\section{LICENSE}

CC BY-NC-SA 4.0

SUBMISSION DATE / POSTED DATE

$10-02-2020$ / 21-02-2020

\section{CITATION}

Narendra, Chaitanya; Mojabi, Puyan (2020): Phaseless Gauss-Newton Inversion for Microwave Imaging. TechRxiv. Preprint. https://doi.org/10.36227/techrxiv.11831295.v1

$\mathrm{DOI}$ 


\title{
Phaseless Gauss-Newton Inversion for Microwave Imaging
}

\author{
Chaitanya Narendra, Student Member, IEEE, and Puyan Mojabi, Member, IEEE
}

\begin{abstract}
A phaseless Gauss-Newton inversion (GNI) algorithm is developed for microwave imaging applications. In contrast to full-data microwave imaging inversion that uses complex (magnitude and phase) scattered field data, the proposed phaseless GNI algorithm inverts phaseless (magnitude-only) total field data. This phaseless Gauss-Newton inversion (PGNI) algorithm is augmented with three different forms of regularization, originally developed for complex GNI. First, we use the standard weighted $L_{2}$ norm total variation multiplicative regularizer which is appropriate when there is no prior information about the object being imaged. We then use two other forms of regularization operators to incorporate prior information about the object being imaged into the PGNI algorithm. The first one, herein referred to as SL-PGNI, incorporates prior information about the expected relative complex permittivity values of the object of interest. The other, referred to as SP-PGNI, incorporates spatial priors (structural information) about the objects being imaged. The use of prior information aims to compensate for the lack of total field phase data. The PGNI, SL-PGNI, and SP-PGNI inversion algorithms are then tested against synthetic and experimental phaseless total field data.
\end{abstract}

Index Terms-Microwave imaging, phaseless (magnitude-only) inversion, inverse scattering, Gauss-Newton inversion (GNI), and regularization.

\section{INTRODUCTION}

$\mathbf{E}$ LECTROMAGNETIC inverse scattering algorithms are used in the microwave imaging (MWI) modality to calculate a quantitative image of the complex dielectric (permittivity) profile in a region of interest (ROI). The ROI, which is commonly referred to as the imaging/investigation domain, contains unknown objects that can often be characterized by analyzing these dielectric reconstructions.

In a microwave imaging system, transmitting antennas successively interrogate the ROI with incident microwave radiation and the resulting total electric (and/or magnetic) fields are measured by receiving antennas on a measurement domain $\mathcal{S}$ outside the ROI. Inverse scattering algorithms then process the measured data, as well as the known incident field data on $\mathcal{S}$ to reconstruct the complex dielectric profile in the ROI. Therefore, these algorithms inherently enable a non-destructive and non-ionizing imaging modality that can be used in many applications such as biomedical imaging, non-destructive evaluation, and remote-sensing [1]-[4].

This work was supported by the Natural Sciences and Engineering Research Council of Canada and the Canada Research Chair Program grants.

C. Narendra, and P. Mojabi are with the Department of Electrical and Computer Engineering, University of Manitoba, Winnipeg, MB, Canada (email: umnarend@myumanitoba.ca).

\section{A. Full Data (Complex) Inversion}

Typically, inverse scattering algorithms use the magnitude and phase (i.e. complex data) of the measured total and incident electric fields to reconstruct the complex permittivity profile within the ROI. (The total and incident fields refer to the measured fields in the presence and absence of the objects being imaged, respectively.) The availability of these complex data enable the calculation of the scattered field data, defined as the difference between the total and incident fields on $\mathcal{S}$. The scattered field data facilitate the imaging process; these data can be thought of as being generated solely by the objects being imaged based on the electromagnetic volume equivalence principle. Therefore, the (complex) scattered field data are processed (inverted) to reconstruct the unknown complex permittivity profile in the ROI. The availability of the scattered field data also enable the use of the so-called scattered field calibration technique [5], which has shown promise to calibrate raw microwave imaging data. Several inverse scattering algorithms (or, simply, inversion algorithms) have been proposed to invert complex scattered field data, e.g., the multiplicatively-regularized contrast source inversion (MR-CSI) [6] and multiplicatively-regularized Gauss-Newton inversion (MR-GNI) [7], [8] algorithms. Due to the use of the multiplicative regularization scheme, these algorithms offer automated adaptive regularization [9], [10]. Furthermore, in conjunction with the scattered field data, these algorithms have also been modified to take into account prior information regarding the ROI. For example, the multiplicative regularization schemes of these two methods have been modified to incorporate prior information about the expected complex permittivity values within the ROI [11], [12].

\section{B. Phaseless Data Inversion - Overview}

Although these state-of-the-art full data inverse scattering algorithms show promise with many applications, their requirement of using both magnitude and phase data can be limiting in some ways. For example, measuring phase information is generally challenging at high frequencies and typically requires expensive equipment, e.g., vector network analyzers, as compared to magnitude-only measurements using affordable power meters. The affordability is, in particular, important depending on the specific imaging application. For example, it may not be reasonable for microwave biomedical imaging to be phaseless due to its required high sensitivity and specificity, however, for some industrial non-destructive testing applications the achievable accuracy from an affordable phaseless microwave imaging system can be sufficient and may be 
desirable. The use of phaseless data has also been considered in another area that is closely related to microwave imaging: near-field antenna measurements and diagnostics. Near-field antenna measurements generally require both magnitude and phase data for near-field to far-field transformation. However, phaseless near-field antenna measurement techniques can be helpful when phase data is not accurate or when near-field measurement system cost must be reduced. In particular, in planar near-field antenna measurements when relatively high probe positioning errors are present, it has been shown that a phaseless approach can outperform the full data (magnitude and phase) approach [13]. This is generally due to the fact that the measured phase data are more sensitive to probe positioning errors as compared to measured magnitude data [13]. For these reasons, phaseless (also known as magnitude-only, amplitude-only or intensity-only) approaches to inverse source and inverse scattering algorithms have been considered. In particular, many phaseless inverse scattering algorithms, such as phaseless MR-CSI [14], [15] have been reported in previous years [16]-[27].

\section{Phaseless Data Inversion - Strategies}

Broadly speaking, two main strategies are used in phaseless data inverse scattering algorithms [22]. The first strategy is a two-step process where the first step retrieves the phase data from measured magnitude-only data, and then standard full data (complex) inverse scattering algorithms invert the retrieved complex scattered field data in the second step [21], [24]. This phaseless strategy in microwave imaging is similar to the iterative Fourier technique [28] in phaseless planar near-field antenna measurements in which the phase data on measurement planes are directly retrieved from two sets of magnitude-only data based on the correlation between the magnitude of the data on two different measurement planes via the iterative use of the plane wave spectrum. The second strategy (the focus of this paper) is a one-step process where the phaseless data are directly inverted to reconstruct the complex permittivity profile of the ROI [14], [16], [18]. This phaseless approach in microwave imaging is similar to the phaseless source reconstruction method in near-field antenna measurements where the phaseless data are directly inverted to reconstruct the equivalent currents of the antenna under test [29]. Herein, we provide a method that falls under the second strategy.

From an information point of view, it is clear that when the phase data are not available, we ideally need to provide the inversion algorithm with some extra information. For example, in phaseless planar near-field antenna measurements, the magnitude data are collected on two measurement planes as opposed to one measurement plane for the case of complex (magnitude and phase) near-field antenna measurements [28], [29]. Although having two planes of measured phaseless data is practical in antenna measurements due to the use of a mechanically scanning probe, this approach is not practical in typical microwave imaging systems because microwave imaging systems typically use co-resident stationary antenna elements [30], [31] to accelerate data collection in order to minimize image artefacts that may be caused from the potential movement of the objects being imaged. In addition, the use of a second measurement domain in phaseless microwave imaging, such as two rings of co-resident antennas, can result in blockage effects. Recently, the use of specialized probes to recover the phase from magnitude-only data has been proposed and experimentally tested with cylindrical dielectric targets [32], [33]. However, it is still worthwhile in phaseless microwave imaging, to investigate other methods to inject information into the inversion algorithm to compensate for the lack of phase data, without having to alter the hardware of existing microwave imaging systems.

In this paper, we first present a phaseless GNI algorithm (there is no explicit phase retrieval step). The phaseless GNI algorithm is then augmented with different forms of multiplicative regularization techniques in order to handle the inherent ill-posedness of the problem and to add prior information to make up for the lack of phase information. To the best of the authors' knowledge, this is the first time that a multiplicatively-regularized phaseless GNI algorithm has been developed. As will be seen, the presented phaseless GNI algorithm starts from a trivial initial guess (relative permittivity of the background) for the ROI, as opposed to a more sophisticated initial guess ${ }^{1}$.

The first regularization scheme used herein is weighted $L_{2}$ norm total variation multiplicative regularization. When the basic phaseless GNI algorithm is augmented with this regularization scheme, we refer to it as PGNI ('p'haseless GNI). We emphasize that PGNI uses the same regularization technique as the phaseless MR-CSI algorithm in [14]. Then, we incorporate prior information into the PGNI algorithm to compensate for the lack of the phase data. First, the PGNI algorithm is augmented with another multiplicative regularizer that incorporates prior information about the expected complex permittivity values (shape and location regularization) in the ROI. We refer the resulting algorithm as SL-PGNI. Finally the PGNI is also augmented with a multiplicative regularizer that takes into account prior structural (spatial prior regularization) information in the ROI. Herein, this algorithm is referred to as SP-PGNI. These regularization methods, originally developed for full data (complex) inversion, are explained in more detail in later sections.

We start by briefly reviewing the full data (complex) MRGNI algorithm in Section II for the sake of completeness. Then, in Section III, the phaseless GNI formulation is developed, along with an overview of the different forms of regularization schemes used to augment this phaseless GNI algorithm. Section IV will explain how the raw experimental data are calibrated prior to the inversion process and Section $\mathrm{V}$ will explain the limitation of our phaseless GNI implementation. Next in Section VI, reconstruction results obtained

\footnotetext{
${ }^{1}$ In contrast to the GNI algorithm, the standard initial guess for CSI relies on the back propagation technique which requires the phase of the data. Therefore, CSI's initial guess is not directly applicable to the phaseless implementation. In [14], an ad hoc procedure based on numerical simulations has been suggested to adapt this initial guess to the phaseless case. In particular, in [14], an ad hoc phase has been assumed for the total field data. Based on this assumption, the scattered data are formed and used for back propagation.
} 
from the inversion of synthetic and experimental data sets are shown and discussed. Finally, the conclusions of this paper are presented in Section VII. A time-dependency of $\exp (j \omega t)$ is considered in this paper along with a $2 \mathrm{D}$ scalar configuration for the imaging setup where the electric field is assumed to be perpendicular to the cross section being imaged.

\section{GNI - A REVIEW}

First, we formally define some terms and then briefly review the standard Gauss-Newton inversion (GNI) algorithm that utilizes full (complex) field data (i.e., magnitude and phase information). Let us denote the incident and total fields by $E^{\text {inc }}$ and $E$ respectively. The difference between the total and incident fields, i.e., the scattered field, is then denoted by $E^{\text {scat }}$. For full data (FD), the inverse scattering problem may then be defined as the minimization of the following data misfit cost functional

$$
\mathcal{C}^{\mathrm{FD}}(\chi)=\eta\left\|F-E^{\mathrm{scat}}(\chi)\right\|^{2}
$$

over $\chi$ where $\|\cdot\|$ is the $L_{2}$ norm taken over the measurement domain $\mathcal{S}$. In addition, $F$ is a complex vector that stores the measured scattered field data on $\mathcal{S}$, and the normalization factor $\eta$ is set to $\|F\|^{-2}$. Moreover, $E^{\text {scat }}(\chi)$ represents the simulated scattered field due to a predicted relative complex permittivity contrast $\chi$. The contrast $\chi(\mathbf{r})$ in the ROI (the unknown we seek) is defined as

$$
\chi(\mathbf{r}) \triangleq \frac{\epsilon(\mathbf{r})-\epsilon_{b}}{\epsilon_{b}}
$$

where $\epsilon(\mathbf{r})$ and is the relative complex permittivity at position $\mathbf{r}$ within the ROI. In addition, $\epsilon_{b}$ is the relative complex permittivity of the background medium. When the GNI algorithm is applied to minimize (1), the contrast at the $n^{\text {th }}$ iteration is updated as $\chi_{n+1}=\chi_{n}+\nu_{n} \Delta \chi_{n}$ where $\nu_{n}$ is the step length (calculated as in [34, Section 9]), and $\Delta \chi_{n}$ is the correction found by solving

$$
\left[\mathbf{J}_{n}^{H} \mathbf{J}_{n}\right] \Delta \chi_{n}=-\mathbf{J}_{n}^{H} d_{n} .
$$

In the above equation, $\mathbf{J}_{n}$ denotes the Jacobian (sensitivity) matrix that represents the derivative of the scattered field data on $S$ with respect to the contrast $\chi$. The subscript $n$ of $\mathbf{J}_{n}$ indicates that this derivative is evaluated at $\chi=\chi_{n}$ and the superscript ' $H$ ' denotes the Hermitian (complex conjugate transpose) operator. Furthermore, $d_{n}$ represents the complex discrepancy vector at the $n^{\text {th }}$ iteration; i.e., $d_{n}=E^{\text {scat }}\left(\chi_{n}\right)-F$. Due to the ill-posedness of the inverse scattering problem, (1) needs to be augmented with a regularization term. For example, at the $n^{\text {th }}$ iteration of the GNI algorithm, $\mathcal{C}^{\mathrm{FD}}(\chi)$ may be multiplicatively regularized as $\mathcal{C}_{n}^{\text {reg }}(\chi)=\mathcal{C}^{\mathrm{FD}}(\chi) \mathcal{C}_{n}^{\mathrm{MR}}(\chi)$ where $\mathcal{C}_{n}^{\mathrm{MR}}$ is the weighted $L_{2}$ norm total variation multiplicative regularizer (MR) given as [7], [8], [35]

$$
\mathcal{C}_{n}^{\mathrm{MR}}(\chi)=\frac{1}{A} \int_{\mathrm{ROI}} \frac{|\nabla \chi(\mathbf{r})|^{2}+\delta_{n}^{2}}{\left|\nabla \chi_{n}(\mathbf{r})\right|^{2}+\delta_{n}^{2}} d \mathbf{s} .
$$

In the above equation, $A$ denotes the area of the ROI, and $\delta_{n}^{2}$ is the steering parameter set to $\mathcal{C}^{\mathrm{FD}}\left(\chi_{n}\right) /(\Delta x \Delta y)$ where $\Delta x \Delta y$ is the area of a single rectangular cell within the discretized ROI; the integration is performed over the area of the ROI.
Applying the GNI algorithm to the multiplicatively-regularized cost functional $\mathcal{C}_{n}^{\text {reg }}$, (3) will change to the following regularized form

$$
\left[\mathbf{J}_{n}^{H} \mathbf{J}_{n}+\beta_{n} \mathcal{L}_{n}\right] \Delta \chi_{n}=-\mathbf{J}_{n}^{H} d_{n}-\beta_{n} \mathcal{L}_{n} \chi_{n}
$$

where $\mathcal{L}_{n}$ is the regularization operator. This operator, when operated on a vector of appropriate size, say $x$, is defined as

$$
\mathcal{L}_{n} x=-\frac{1}{A} \nabla \cdot\left(\frac{1}{\left|\nabla \chi_{n}(\mathbf{r})\right|^{2}+\delta_{n}^{2}} \nabla x\right) .
$$

In the above expression, ' $\nabla \cdot$ ' and ' $\nabla$ ' denote the divergence and gradient operators, respectively. Finally, the weight of this operator in (5), i.e., $\beta_{n}$, is $\mathcal{C}^{\mathrm{FD}}\left(\chi_{n}\right) / \eta$. Herein, we refer to this algorithm as the MR-GNI algorithm. We emphasize that the abbreviation MR-GNI, when used in this paper, implies the use of full (complex) data. This completes our review of the MR-GNI algorithm which was mainly based on [7], [8], [35]. It is instructive to note that the right hand side of (5) consists of two components. The first one represents the (negative) gradient of the data misfit cost functional $\mathcal{C}^{\mathrm{FD}}(\chi)$, thus, helping the algorithm extract the information within $F$ to reconstruct $\chi$. The second term represents the (negative) gradient of the regularization term $\mathcal{C}_{n}^{\mathrm{MR}}$ which helps the algorithm stabilize the inversion process and apply some edge-preserving operations. The relative weight of these two gradients are controlled by $\beta_{n}$ which comes directly from the multiplicative nature of the regularized cost functional $\mathcal{C}^{\text {reg }}$. Finally, the left hand side of (5) represents the operation of the Hessian matrix ${ }^{2}$ on $\Delta \chi_{n}$. As expected, the Hessian consists of two parts as well: one for the data misfit cost functional, and the other for the regularization term.

\section{Phaseless GNI}

\section{A. Phaseless Data Misfit Cost Functional}

Herein, we begin to discuss the phaseless GNI algorithm. The lack of phase information prevents the scattered field data from being calculated. Therefore, the data to be inverted for the phaseless GNI algorithm will be the magnitude of the total field data. Herein the magnitude of the total field data is stored in the vector $M$. We then form the phaseless data misfit cost functional as

$$
\mathcal{C}(\chi)=\zeta\left\|M^{2}-|E(\chi)|^{2}\right\|^{2}
$$

where $|E(\chi)|$ denotes the magnitude of the simulated total field data due to a predicted contrast $\chi$. In addition, $\zeta$ is the normalization factor that has been set to

$$
\zeta=\left\|M^{2}-\left|E^{\mathrm{inc}}\right|^{2}\right\|^{-2} .
$$

Similar to the full data (complex) MR-GNI algorithm, the contrast at the $n^{\text {th }}$ iteration is updated according to $\chi_{n+1}=\chi_{n}+\nu_{n} \Delta \chi_{n}$ (where $\nu_{n}$ was calculated using the

${ }^{2}$ In the GNI algorithm, the Hessian matrix is obtained by ignoring the second derivative of $E^{\text {scat }}$ with respect to $\chi$, e.g., see [36]. In other words, the GNI algorithm applies Newton optimization to the regularized data misfit cost functional. However, the second-order derivative of the cost functional is calculated under the approximation that the second derivative of the scattered field (not the cost functional) with respect to $\chi$ is zero. 
methods in [34, Section 9]). As derived in the Appendix, the correction $\Delta \chi_{n}$ at the $n^{\text {th }}$ iteration is then found from

$$
\begin{aligned}
& {\left[2 \mathbf{J}_{n}^{H} \operatorname{diag}\left(2\left|E\left(\chi_{n}\right)\right|^{2}-M^{2}\right) \mathbf{J}_{n}\right] \Delta \chi_{n}=} \\
& \quad-2 \mathbf{J}_{n}^{H}\left[E\left(\chi_{n}\right) \odot\left(\left|E\left(\chi_{n}\right)\right|^{2}-M^{2}\right)\right]
\end{aligned}
$$

where 'diag' represents the diagonal operator that turns a vector into a diagonal matrix, and $\odot$ denotes the elementwise (Hadamard product) of two vectors of the same size. Note that the Jacobian matrix $\mathbf{J}_{n}$ used in (9) is the same as that used in (3). This may come as a surprise since in the full data (complex) GNI, $\mathbf{J}_{n}$ represents the derivative of the scattered field with respect to the contrast at the $n^{\text {th }}$ iteration of the algorithm; and in phaseless GNI $\mathbf{J}_{n}$ should represent the derivative of the total field with respect to the contrast at the $n^{\text {th }}$ iteration. However, since the total field is the summation of the incident and scattered fields, and noting that the incident field does not depend on the contrast, the derivative of the total field with respect to the contrast is the same as the derivative of the scattered field with respect to the contrast; i.e., $\frac{\partial E^{\text {scat }}}{\partial \chi}=\frac{\partial E}{\partial \chi}$. Also, note that in (9), $E\left(\chi_{n}\right)$ represents the vector that contains the simulated total field on the measurement domain $\mathcal{S}$ due to the predicated contrast $\chi_{n}$. As can be seen in (9), both magnitude and phase of $E\left(\chi_{n}\right)$ have been used. This is not contradictory to phaseless inversion since $E\left(\chi_{n}\right)$ represents the simulated total field data due to the predicted contrast $\chi$, and not the phaseless measured data. Finally, we note that in all the examples shown herein, we use a trivial initial guess, $\chi=0$, to start the phaseless GNI algorithm.

\section{B. Regularization}

Similar to the full data (complex) GNI algorithm that required regularization, the phaseless GNI algorithm also requires the regularization of its cost functional (7). Note that regularization is typically performed on the contrast $\chi$; therefore, the regularization operators for the phaseless problem can be the same as the complex problem. Herein, we consider three types of multiplicative regularization schemes. The first is the weighted $L_{2}$ norm total variation multiplicative regularizer given in (4), which does not assume any particular prior information about the contrast profile in the ROI. The second is developed for shape and location reconstruction [11], [12]. This regularization assumes prior information about the complex permittivity values (thus, the contrast values) within the ROI. The problem then becomes one of finding the shape and location of the dielectric scatterers within the ROI. The third assumes prior information about the shape (structural information) of the target's regions of identical relative permittivity [37], [38]. If this spatial prior regularizer is used, the problem becomes one of reconstructing the appropriate complex permittivity values within the known regions of the ROI. When the phaseless GNI algorithm is used with each of these three regularization schemes, we refer to the resulting three different regularized algorithms as PGNI, SL-PGNI and SP-PGNI, respectively.

\section{PGNI Algorithm}

Similar to the complex GNI algorithm, we multiplicatively regularize the phaseless data misfit cost functional as

$$
\mathcal{C}_{n}^{\mathrm{reg}}(\chi)=\mathcal{C}(\chi) \mathcal{C}_{n}^{\mathrm{MR}}(\chi) .
$$

Applying the GNI algorithm to this regularized phaseless cost functional, (9) will turn into the following regularized form

$$
\begin{array}{r}
{\left[2 \mathbf{J}_{n}^{H} \operatorname{diag}\left(2\left|E\left(\chi_{n}\right)\right|^{2}-M^{2}\right) \mathbf{J}_{n}+\tau_{n} \mathcal{L}_{n}\right] \Delta \chi_{n}=} \\
\quad-2 \mathbf{J}_{n}^{H}\left[E\left(\chi_{n}\right) \odot\left(\left|E\left(\chi_{n}\right)\right|^{2}-M^{2}\right)\right]-\tau_{n} \mathcal{L}_{n} \chi_{n}
\end{array}
$$

where $\tau_{n}$ is $\mathcal{C}\left(\chi_{n}\right) / \zeta$. Herein, we refer to this phaseless algorithm as the PGNI algorithm. Note that the PGNI algorithm does not use any particular prior information about the ROI, and can therefore be considered as a blind phaseless inversion algorithm.

\section{SL-PGNI Algorithm}

This regularization scheme incorporates prior information about the expected contrast values within the ROI. Therefore, it is mainly used to reconstruct the shape and location of the dielectric scatterers within the ROI. To this end, we augment (10) with an extra regularization term

$$
\mathcal{C}_{n}^{\mathrm{SL}, \mathrm{reg}}(\chi)=\mathcal{C}(\chi) \mathcal{C}_{n}^{\mathrm{MR}}(\chi) \mathcal{C}_{n}^{\mathrm{SL}}(\chi)
$$

where the superscript 'SL' notes the suitability of this regularizer for shape and location reconstruction. This regularization term is given as [11], [12]

$$
\mathcal{C}_{n}^{\mathrm{SL}}(\chi)=\frac{1}{A} \int_{\mathrm{ROI}} \prod_{\ell=1}^{L} \frac{\left|\chi(\mathbf{r})-\chi_{\ell}\right|^{2}+\alpha_{n}^{2}}{\left|\chi_{n}(\mathbf{r})-\chi_{\ell}\right|^{2}+\alpha_{n}^{2}} d \mathbf{s}
$$

where $\chi_{\ell}$ for $\ell=1$ to $L$ denotes the expected values of the complex contrast, and $\prod_{\ell=1}^{L}$ denotes the product of $L$ different functions. The simplest form of this regularization term is binary regularization in which we are dealing with two values of the contrast: $\chi_{1}=0$ which is for the background medium, and $\chi_{2}$ which represents the expected contrast value of the scatterer. We emphasize that the given prior information $\chi_{\ell}$ contains no knowledge about the shape and location of the scatterers. In addition, $\alpha_{n}^{2}$ is the steering parameter which is set to $\alpha_{n}^{2}=\mathcal{C}\left(\chi_{n}\right)$. Applying the GNI algorithm to (12) will change (11) to

$$
\begin{aligned}
{\left[2 \mathbf { J } _ { n } ^ { H } \operatorname { d i a g } \left(2\left|E_{n}\right|^{2}\right.\right.} & \left.\left.-M^{2}\right) \mathbf{J}_{n}+\tau_{n} \mathcal{L}_{n}+\tau_{n} \sum_{\ell=1}^{L} \mathcal{R}_{n, \ell}\right] \Delta \chi_{n} \\
= & -2 \mathbf{J}_{n}^{H}\left[E_{n} \odot\left(\left|E_{n}\right|^{2}-M^{2}\right)\right]-\tau_{n} \mathcal{L}_{n} \chi_{n} \\
& -\tau_{n} \sum_{\ell=1}^{L} \mathcal{R}_{n, \ell}\left(\chi_{n}-\chi_{\ell}\right)
\end{aligned}
$$

where the regularization operators $\mathcal{R}_{n, \ell}$ when operating on a vector $x$ of appropriate size are given as [12]

$$
\mathcal{R}_{n, \ell} x=\frac{1}{A} \operatorname{diag}\left(\frac{1}{\left|\chi_{n}(\mathbf{r})-\chi_{\ell}\right|^{2}+\alpha_{n}^{2}}\right) x .
$$

We refer to this phaseless GNI algorithm with prior expected contrast values as SL-PGNI. 


\section{E. SP-PGNI Algorithm}

In contrast to the above shape and location regularization scheme which assumed prior information about the expected complex permittivity values, we now consider a spatial prior regularization scheme which assumes prior spatial (or structural) information in the ROI without making any assumptions regarding their complex permittivity values. In this prior information approach, the shapes of the regions having identical permittivity are assumed to be known ${ }^{3}$. If this regularization scheme is used, the resulting inverse scattering algorithm attempts to reconstruct the relative complex permittivity values within these regions. Intuitively, one can think of this regularizer as reducing the amount of $\chi$ variables in the ROI, because there are fewer regions of identical permittivity than the original number of discrete cells in the ROI. To this end, (10) is augmented with an extra regularization term

$$
\mathcal{C}_{n}^{\mathrm{SP} \text {,reg }}(\chi)=\mathcal{C}(\chi) \mathcal{C}_{n}^{\mathrm{MR}}(\chi) \mathcal{C}_{n}^{\mathrm{SP}}(\chi)
$$

where the superscript ' $\mathrm{SP}$ ' denotes the spatial prior information given to the regularizer. This regularization term is given as [37], [38]

$$
\mathcal{C}_{n}^{\mathrm{SP}}(\chi)=\frac{\|p \odot(\mathbf{A} \chi)\|^{2}+\gamma_{n}^{2}}{\left\|p \odot\left(\mathbf{A} \chi_{n}\right)\right\|^{2}+\gamma_{n}^{2}}
$$

In $\mathcal{C}_{n}^{\mathrm{SP}}(\chi), \mathbf{A}$ is a sparse matrix consisting of only zeros and \pm 1 . Its purpose is to enforce equality between contrast values in the specified regions of identical permittivity. In other words, the prior structural information of the ROI is stored in the matrix $\mathbf{A}$ via several 0 and \pm 1 elements. (This choice of A was also utilized in [40], [41] in the form of an additive regularization scheme for complex GNI.) The probability vector $p$ contains elements $0 \leq p_{i} \leq 1$. In this work, $p_{i}$ has been set to 1 for all $i$ 's. Finally, $\gamma_{n}^{2}$ is a steering parameter chosen to be $\gamma_{n}^{2}=\mathcal{C}\left(\chi_{n}\right) N$ where $N$ is the length of the vector $\chi[37$, Section D]. Applying the GNI algorithm to (16) will result in

$$
\begin{aligned}
& {\left[2 \mathbf{J}_{n}^{H} \operatorname{diag}\left(2\left|E_{n}\right|^{2}-M^{2}\right) \mathbf{J}_{n}+\tau_{n} \mathcal{L}_{n}+\tau_{n} \mathcal{S}_{n}\right] \Delta \chi_{n}=} \\
& \quad-2 \mathbf{J}_{n}^{H}\left[E_{n} \odot\left(\left|E_{n}\right|^{2}-M^{2}\right)\right]-\tau_{n} \mathcal{L}_{n} \chi_{n}-\tau_{n} \mathcal{S}_{n} \chi_{n}
\end{aligned}
$$

where the operator $\mathcal{S}_{n}$ acting on a vector $x$ of appropriate size is

$$
\mathcal{S}_{n} x=\frac{1}{\left\|p \odot\left(\mathbf{A} \chi_{n}\right)\right\|^{2}+\gamma_{n}^{2}} \mathbf{A}^{H}(p \odot(p \odot(\mathbf{A} x))) .
$$

Herein, we refer to this phaseless GNI algorithm with prior spatial information as SP-PGNI.

\section{CAlibration of The Experimental Data}

\section{A. Classification - Review}

In microwave imaging, there will always be some discrepancy between the actual measurement environment, and the numerical model used in the inversion algorithm. To alleviate

\footnotetext{
${ }^{3}$ For example, in a combined magnetic resonance imaging (MRI) and microwave imaging system, the high resolution structural information obtained from the MRI can be given as spatial prior information to the microwave imaging system [39].
}

these discrepancies, so-called data calibration techniques such as the scattered field or the incident field calibration methods [5] are used. ${ }^{4}$ For phaseless inversion, the scattered field data are not available, and therefore, the scattered field calibration technique cannot be used. Therefore, we decided to use the incident field calibration technique for our experimental data. There are at least two ways that incident field calibration can be applied. In the first method, a simulated incident field, such as a zeroth-order Hankel function of the second kind is assumed for the incident field, and then complex calibration coefficients are found to reduce the discrepancy between the simulated and measured incident field data. These calibration coefficients are then used to modify the raw experimental data. The second method is a more general way, which is based on the so-called source reconstruction method (SRM). ${ }^{5}$

\section{B. SRM Calibration}

In the SRM-based calibration method, the SRM is used to find equivalent surface current distributions for the transmitting antennas that can also generate the measured incident field data. Once these equivalent currents are found, they can be used in the GNI algorithm to represent the actual antennas. Since these equivalent currents are associated with the raw incident field data, the raw phaseless total field data are directly given to the inversion algorithm to be inverted. Therefore, as opposed to the previous calibration methods, the SRMbased calibration method makes it possible to invert the raw experimental data directly.

Herein, we have used the second method; i.e., the SRM for incident field calibration. The details of this calibration method can be found in [44]. To this end, we have utilized the SRM to replace each transmitting antenna with its equivalent currents. That is, for example, if 24 antennas are present in a microwave imaging setup, we have replaced them with 24 sets of equivalent currents. We have used both the magnitude and phase of the measured incident field. Thus, the inverse source problem associated with the SRM becomes a linear inverse source problem. Due to the fact that the L-curve method is particularly suited for linear ill-posed problems [10], [45], we have used the L-curve method to choose an appropriate solution when performing SRM. ${ }^{6}$

\section{LIMITATION}

Note that the above calibration method requires the magnitude and phase of the incident field data (not the phaseless total field data). The presence of the phase data for the incident field is not a bad assumption since it can be regarded as part of system characterization (antenna characterization) prior to

\footnotetext{
${ }^{4}$ Similar to the calibration object in radar cross section measurements, the scattered field calibration technique in microwave imaging uses an object, such as a metallic cylinder [42], for which the complex scattered fields are analytically known. These analytical expressions and the measured scattered field data are then compared to construct complex-valued calibration coefficients, which will then used to calibrate the actual measured data.

${ }^{5}$ The source reconstruction method is, in fact, an electromagnetic inverse source algorithm [43].

${ }^{6}$ The L-curve method requires determining the knee point of the L-curve; in our implementation the knee point is chosen in an ad hoc manner.
} 
performing imaging. This assumption is also present in many other phaseless microwave imaging algorithms [14], [15], [21], [22], [24]. In all of these algorithms, the total field data, but not the incident field data, are assumed to be phaseless. If this assumption is not made, we would have to use a phaseless SRM algorithm, e.g., see [29], to characterize the antennas using measured phaseless incident fields. Once the equivalent currents of the antennas are obtained using phaseless incident fields, it can be used in the phaseless GNI algorithm similar to the above. Therefore, in summary, similar to other phaseless microwave imaging algorithms, this paper assumes phaseless total field data but considers complex incident field data.

\section{RESULTS}

Herein, we show synthetic and experimental results to evaluate the performance of our phaseless GNI algorithms. First, the PGNI, SL-PGNI, and SP-PGNI algorithms are used to reconstruct images of a pair of lossy concentric squares in the ROI using synthetically generated data. Next, experimental data collected from the Institut Fresnel in France [46] are used to validate the PGNI, SL-PGNI, and SP-PGNI algorithms. Because the experimental data from Institut Fresnel are from lossless targets, we then consider experimental data obtained from a skinless bovine leg [35] to evaluate the performance of the PGNI, SL-PGNI, and SP-PGNI algorithms against a lossy object. In addition to lossy versus lossless objects, there are two other differences between these two data sets: (I) the Fresnel data sets use a mechanical scanning probe to collect the data whereas the bovine leg data are collected by 24 coresident dipole antennas; (II) the background medium in the Fresnel data sets is air whereas the background medium in the bovine leg data set is salty water. ${ }^{7}$

Since these experimental data sets all contained measured magnitude and phase data, we removed the phase of the measured total field data, and only worked with the measured magnitude-only total field data. Finally, we note that the iterative nature of the phaseless inversion algorithms means that a stopping condition is necessary. In this work, we stopped the algorithm when $\mathcal{C}\left(\chi_{n}\right)$ decreased below $10^{-3}$ or when the change in the reconstructed contrast was smaller than $10^{-4}$ after two consecutive iterations.

\section{A. Synthetic Concentric Squares Data Set}

To validate our phaseless GNI algorithms, synthetic data at $4 \mathrm{GHz}$ were created from a pair of concentric dielectric squares with a background of free-space $\left(\epsilon_{r}=1\right)$. The relative complex permittivities of the outer and inner squares were $\epsilon_{r}=1.3-0.4 j$ and $\epsilon_{r}=1.6-0.2 j$, respectively, see Figure 1(a)-(b). For phaseless data collection, 36 transceivers were located on a circle of radius $76 \mathrm{~cm}$ around the origin. (The transceivers are assumed to be infinite line sources,

\footnotetext{
${ }^{7}$ To reduce unwanted reflections, the Fresnel data sets were collected in an anechoic chambers with absorbers. On the other hand, for the bovine leg data, salt has been added to water to make it lossy, thus reducing the reflections from the imaging chamber wall. This is necessary since the imaging algorithm assumes a free space Green's function that does not take into account the reflections from the walls of the imaging chamber.
}

thus, they are numerically modelled by zeroth-order Hankel functions of the second kind.) The ROI was discretized into 43 by 43 square elements and was $166 \mathrm{~mm}$ by $166 \mathrm{~mm}$ in size. The inversion was done at a single frequency of $4 \mathrm{GHz}{ }^{8}$ The results from attempting to reconstruct the lossy concentric squares from phaseless total field data using the PGNI, SLPGNI, and SP-PGNI algorithms are shown in Figure 1(c)-(h). As noted earlier, the PGNI is blind phaseless inversion (no prior information is given to the algorithm). In the case of SLPGNI, the phaseless inversion algorithm was provided with the true three contrast values as prior information, i.e., the contrast value of the background medium and the contrasts of the two squares. The SL-PGNI then reconstructs the shape and location of the objects being imaged. Finally, in the case of the SPPGNI, the spatial map of the target (i.e. the regions of identical permittivity) was provided to the phaseless inversion algorithm as prior structural information. The SP-PGNI then reconstructs the complex permittivity values in these spatial regions. As can be seen, the SL-PGNI and SP-PGNI reconstructions were more accurate than that of the PGNI algorithm. This is to be expected as the latter two algorithms include more information about the objects being imaged whereas the PGNI algorithm is a blind inversion.

\section{B. Experimental FoamDielIntTM Data Set}

To study the performance the phaseless GNI algorithms with experimental data, we consider the measured data provided by the Institut Fresnel in France [46]. The phase of the measured total field was disregarded and only its magnitude data were used. The first target consists of two dielectric cylinders, one inside the other, and the background is air. The inner-most cylinder is $31 \mathrm{~mm}$ in diameter with a relative permittivity of $\epsilon_{r}=3 \pm 0.3$, and is slightly offset from being concentric with the outer cylinder by $5 \mathrm{~mm}$ in the $x$-direction. The outer cylinder has a relative permittivity of $\epsilon_{r}=1.45 \pm 0.15$ and a diameter of $80 \mathrm{~mm}$. This target is referred to as FoamDielIntTM by [46] and is shown in Figure 2(a). Note that the imaginary parts of the complex permittivities of these cylinders are zero, and therefore, the objects being imaged are lossless. The experimental dataset used for the FoamDielIntTM inversions included eight transmitters and 241 receivers for each transmitter. The transmitters and receivers were located $1.67 \mathrm{~m}$ from the centre of the ROI [46] and the ROI was discretized into 65 by 65 elements and was $150 \mathrm{~mm}$ by $150 \mathrm{~mm}$ in size. The frequency of inversion was $2 \mathrm{GHz}$.

In Figure 2(b)-(c) the result of inverting the FoamDielIntTM data with PGNI are shown and the reconstructed overall shape and permittivities are reasonable; however, they are not very accurate. For example, the reconstructed size of the inner cylinder is greater than the actual one, and its reconstructed permittivity is smaller than its true permittivity. In addition, there is a small imaginary part present in the reconstructed permittivity. In Figure 2(d)-(e), the reconstructed permittivity using SL-PGNI is shown. In the SL-PGNI algorithm, the relative permittivities of the cylinders are assumed to be known

\footnotetext{
${ }^{8}$ The synthetic data were created on a different grid to avoid the so-called inverse crime.
} 


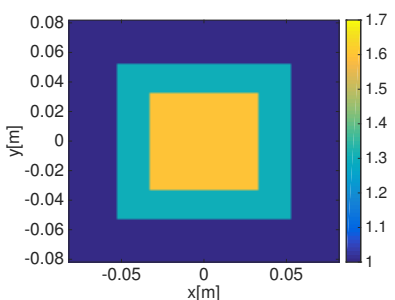

(a)

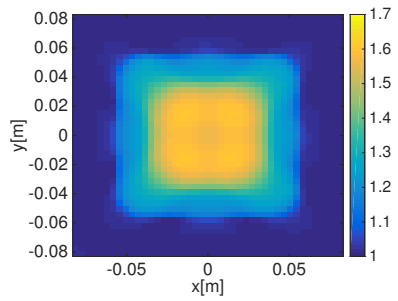

(c)

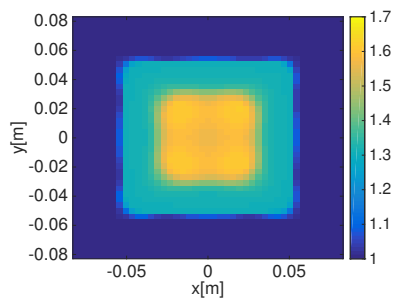

(e)

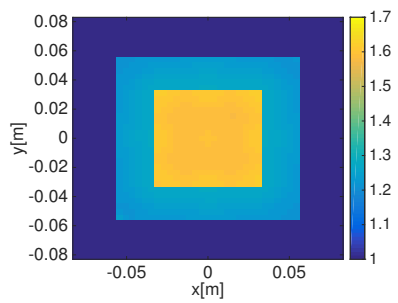

(g) (b)

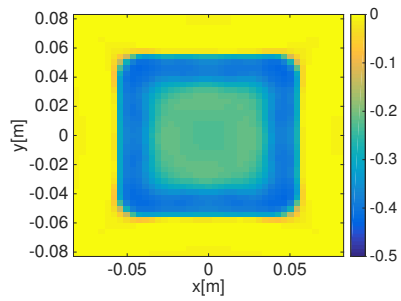

(d)

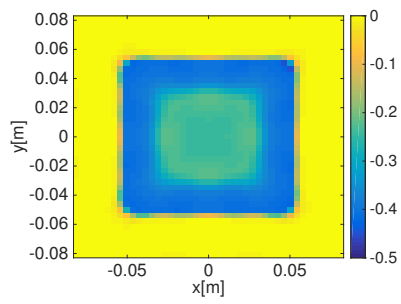

(f)

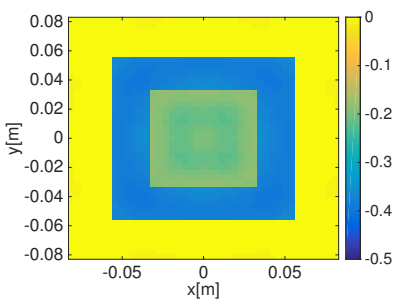

(h)

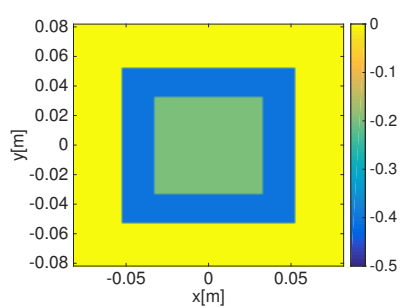

Fig. 1. The real (left) and imaginary (right) parts of the reconstructed relative permittivity from the inversion of the synthetically generated phaseless total field data from two lossy concentric squares (first row) at $4 \mathrm{GHz}$ using the PGNI (second row), SL-PGNI (third row), and SP-PGNI (fourth row) algorithms, respectively.

and are given as prior information to the phaseless inversion algorithm. As a result, the SL-PGNI algorithm reconstructs the shape and location of the target more accurately compared to the blind phaseless inversion algorithm (PGNI). Similar to the PGNI reconstruction, the SL-PGNI reconstruction also shows small imaginary parts in the complex permittivity (i.e., some small loss). ${ }^{9}$

\footnotetext{
${ }^{9}$ The fact that there are small imaginary parts in the reconstructed permittivity using the SL-PGNI algorithm may come as a surprise. This is due to the fact that the SL-PGNI gets the value of the permittivity as the prior information. In this particular inversion, we have provided the SL-PGNI algorithm with the relative permittivity of 3 and 1.45 (and, of course the relative permittivity of 1 for the background). That is, the imaginary parts of the relative complex permittivities in the prior information were zero. However, these values are only enforced as soft regularization in the sense that the inversion algorithm will favour these values but still has the chance to not completely enforce them.
}

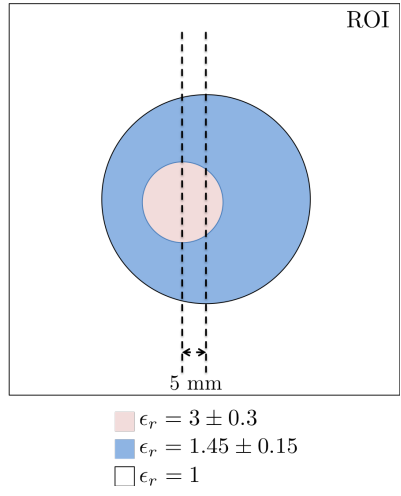

(a)

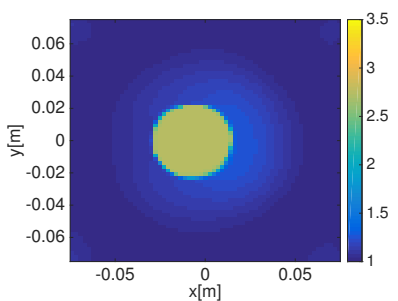

(b)

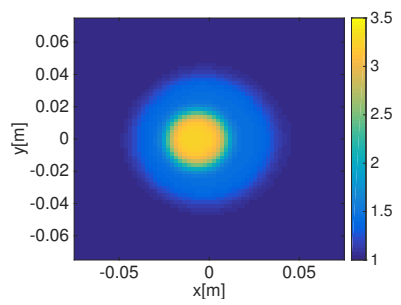

(d)

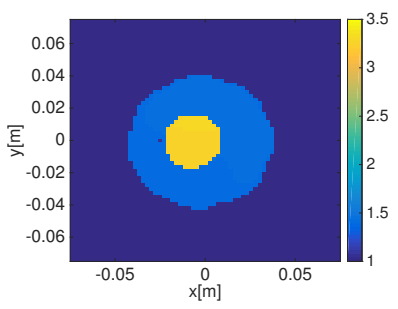

(f)

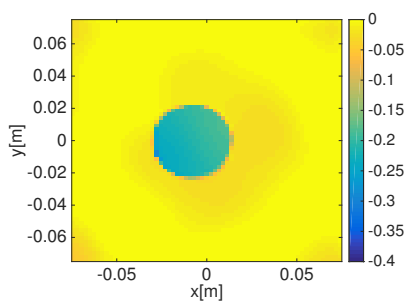

(c)

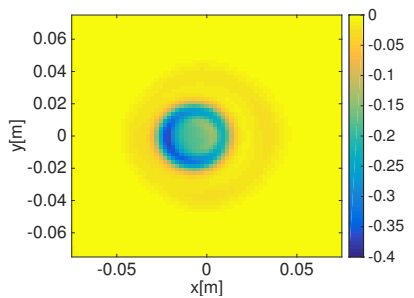

(e)

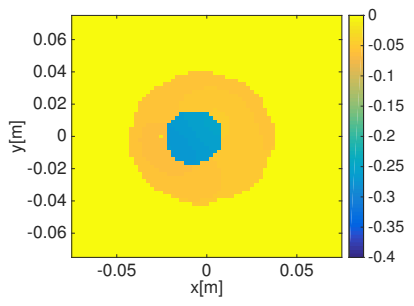

(g)
Fig. 2. The FoamDielIntTM Fresnel data [46], without the phase of the measured total field, are inverted. An illustration of the true target is shown (top row) before showing the reconstructed permittivity results for a single frequency of $2 \mathrm{GHz}$ from the PGNI (second row), SL-PGNI (third row), and SP-PGNI (fourth row) algorithms. The real (left) and imaginary (right) parts of the reconstructed relative permittivity from the inversion are reported.

We now invert these phaseless measured data using the SP-PGNI algorithm. To this end, we need to give the structural information of this target as prior information to SPPGNI. Ideally, this spatial prior information should come from a higher resolution imaging modality such as magnetic resonance imaging (MRI). Once high resolution structural information is given to SP-PGNI, this inversion algorithm will aim to find the complex permittivity values in different regions from phaseless data. However, since MRI data were not available for this target, we use a different method to 


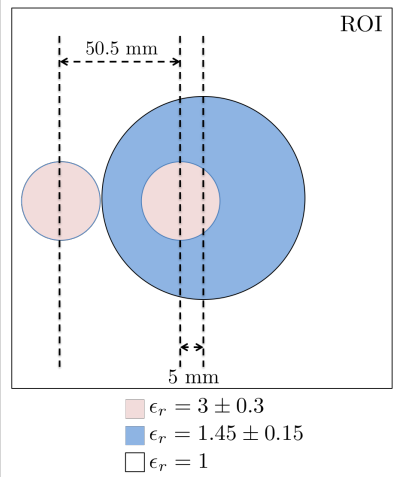

(a)

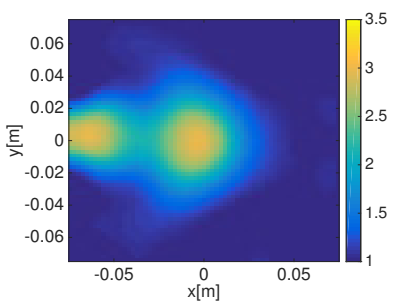

(b)

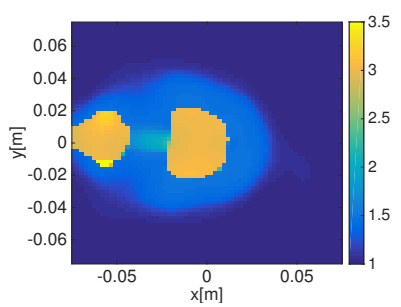

(d)

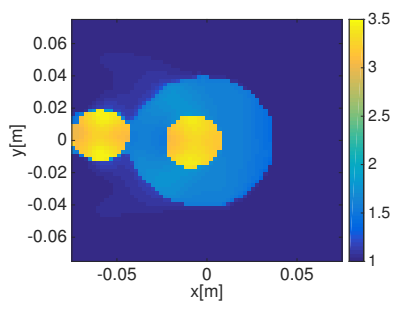

(f)

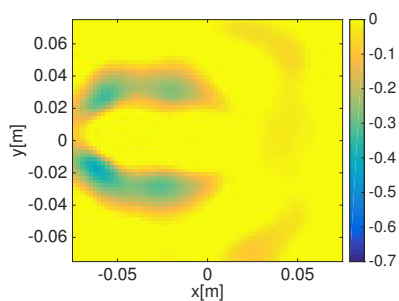

(c)

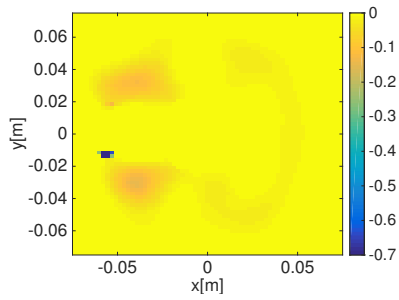

(e)

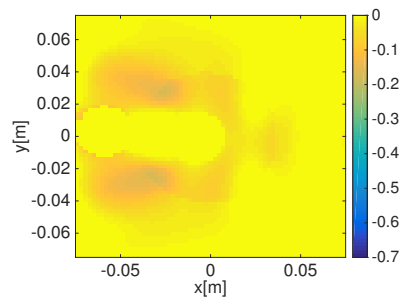

(g)
Fig. 3. The FoamTwinDielTM dataset [46] without the phase of the measured total field, are inverted. An illustration of the true target (top row) is presented. The real (left) and imaginary (right) parts of the reconstructed relative permittivity from inversions at $4 \mathrm{GHz}$ using the PGNI (second row), SL-PGNI (third row), and SP-PGNI (fourth row) algorithms are shown.

create the spatial prior map; we used the full data (complex) MR-GNI algorithm to invert the data set at $4 \mathrm{GHz}, 6 \mathrm{GHz}$ and $10 \mathrm{GHz}$ simultaneously. The achieved inversion result is then used to create spatial priors for this target. (Note that we do not provide any prior information regarding the complex permittivity values for SP-PGNI.) These spatial priors are then given to the SP-PGNI algorithm to find the complex permittivity at these regions. The reconstruction results using the SP-PGNI algorithm are shown in Figure 2(f)-(g).

\section{Experimental FoamTwinDielTM Data Set}

Next, inversion results for the FoamTwinDielTM experimental data from the Institut Fresnel are shown [46]. (Note that we once again disregard the phase and invert just the magnitude of the total field data.) For this experiment, the target is similar to the FoamDielIntTM case, except there is another small cylinder with the same dielectric properties and size as the inner-most cylinder external to the outer cylinder. The two small cylinders are $55.5 \mathrm{~mm}$ apart. An illustration of the FoamTwinDielTM target is shown in Figure 3(a). The data collection for this test included 18 transmitters with 241 receivers each. Once again, the transmitters and receivers are located on a circle of radius $1.67 \mathrm{~m}$ and the ROI was discretized into a 65 by 65 grid with side lengths of $150 \mathrm{~mm}$ each. The results of the inversion using the PGNI, SL-PGNI and SP-PGNI algorithms are shown in Figure 3(b)-(g) for a frequency of $4 \mathrm{GHz}$. (Similar to the previous example, the spatial priors for the SP-PGNI algorithm were obtained through a multi-frequency full-data MR-GNI algorithm.) In addition, similar to the previous example, the relative permittivity values given as prior information to the SL-PGNI algorithm are 3, 1.45 , and 1. It can be observed once again that adding more information through more sophisticated regularization schemes with the SL-PGNI and SP-PGNI algorithms enables sharper reconstructions, with more evidence of the larger cylinder. Comparing the reconstructions from the SL-PGNI and SPPGNI algorithms shows that for the SL-PGNI algorithm, the permittivities are smooth but the shape of the reconstructed cylinders is not completely circular. In contrast the SP-PGNI algorithm has a much more accurate shape, but the permittivity is not as smooth overall for the small dielectric cylinders as the SL-PGNI algorithm. This result is expected as both algorithms do well reconstructing what they have been informed is prior information.

\section{Experimental Skinless Bovine Leg Data Set}

In order to test the phaseless GNI algorithms with a lossy target and background medium in the ROI, we use the experimental data collected from a skinless bovine leg in a salt-water medium using 24 co-resident dipole antennas [35]. (Similar to the previous cases, we ignore the phase of the measured total field data, and invert only its magnitude data.) The ROI is a square discretized into a 50 by 50 grid with a total length of $120 \mathrm{~mm}$. The data was collected at $0.8 \mathrm{GHz}$ with the relative complex permittivity of the background being $\epsilon_{r}=76-14 j$. The expected relative permittivity values of the bone (centremost region of the bovine leg) is $\epsilon_{r}=26-8 j$, and flexor (region around bone) is $\epsilon_{r}=54-18 j$ [35]. Prior to showing the phaseless inversions, let us take a look at the inversion of the full data (magnitude and phase) as shown in Figure 4(a)(b). ${ }^{10}$

\footnotetext{
${ }^{10}$ Herein, for the full data (complex) case, we have also used the SRMbased incident field calibration method. We note that the inversion of these full data using the scattered field calibration method, as shown in [35], seems to be better than the inversion shown in Figure 4(a)-(b) (and, also shown in [44]). However, since the use of the scattered field calibration method was not possible for the phaseless case, we have also calibrated the full data using the SRM-based incident field calibration method for consistency.
} 


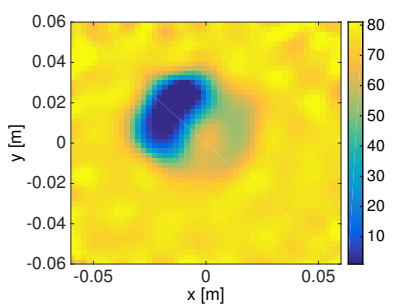

(a)

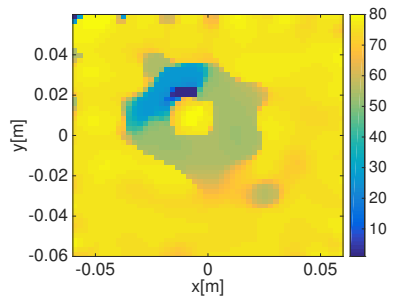

(c)

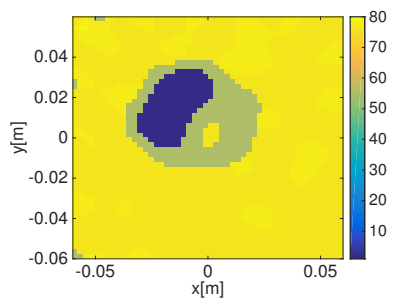

(e)

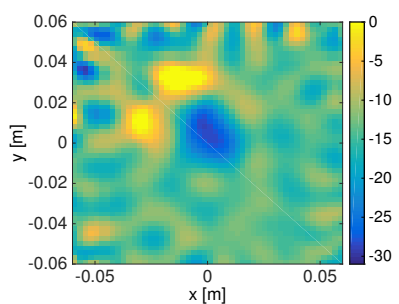

(b)

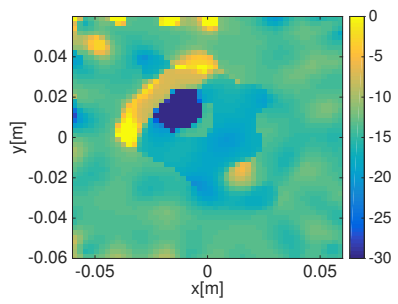

(d)

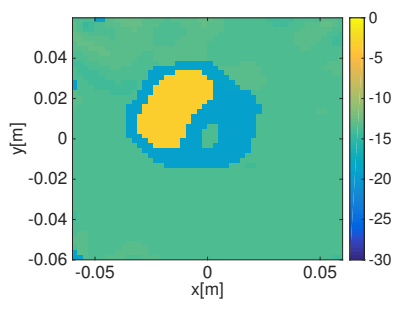

(f)
Fig. 4. Experimental inversion results for a skinless bovine leg in a salt water medium [35]. The real (left) and imaginary (right) parts of the reconstructed relative permittivity at $0.8 \mathrm{GHz}$ using the full data (complex) MR-GNI (first row), and the phaseless SL-PGNI (second row) and SP-PGNI (third row) algorithms are shown.

For this lossy experimental data set, the blind phaseless inversion algorithm (i.e., the PGNI algorithm) failed to provide any meaningful reconstruction; thus, it is not shown here. We then tried the phaseless inversion algorithms incorporating prior information; i.e., the SL-PGNI and SP-PGNI algorithms; the reconstruction results using these phaseless algorithms are shown in Figure 4(c)-(f). It can be seen that the SL-PGNI algorithm performed poorly when compared with the SPPGNI algorithm. This is an indication that the spatial prior information may have been more useful in reconstructing the true target than the prior knowledge of expected permittivities. Also, in the reconstruction process, we have limited the variation of the allowed relative complex permittivity as follows: $1 \leq \operatorname{Re}\left(\epsilon_{r}\right) \leq 80$ and $-30 \leq \operatorname{Im}\left(\epsilon_{r}\right) \leq 0$. Due to this enforcement, the reconstructed permittivity of the bone using the SP-PGNI method shown in Figure 4(e)-(f) looks very uniform, but is mistakenly similar to free-space and not bone (i.e., $\epsilon_{r}=26-8 j$ ) as it should be. In addition, the phaseless inversion of the skinless bovine leg data set had another challenge: the steering parameters of the phaseless inversion algorithms (i.e., $\delta_{n}^{2}, \alpha_{n}^{2}$, and $\gamma_{n}^{2}$ ) had to be modified

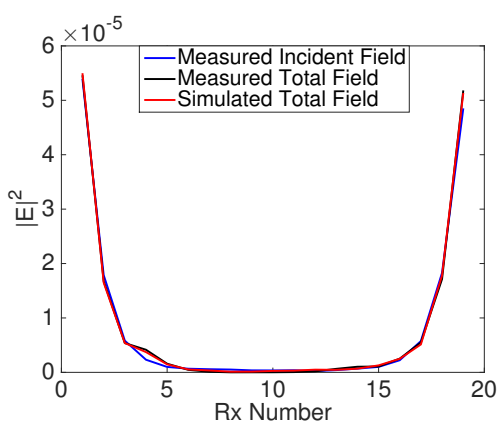

(a)

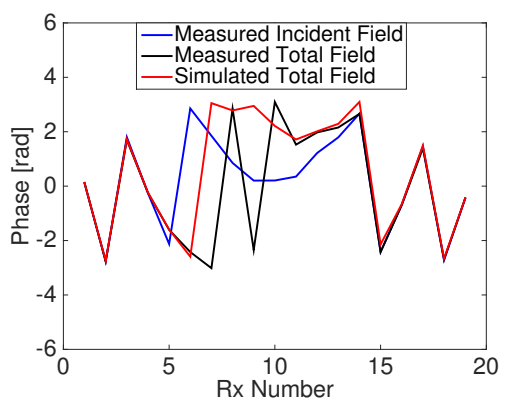

(b)

Fig. 5. The squared magnitudes of the measured total field, the measured incident field, and simulated total field from the SL-PGNI algorithm [left] are shown to be very similar showing the lack of information present about the bovine target in the measured magnitude data. The phase distributions are then shown [right] to be more varied. This is shown for one transmitter but the same plots are similar for all transmitters.

for this particular example to work. ${ }^{11}$ That is, the phaseless inversion of this data set was more sensitive to the overall regularization weight as compared to the other cases. This was perhaps due to the lossy nature of the complex permittivities which further reduces the information content of the measured data.

It is instructive to investigate the information content available for the phaseless inversion in this skinless bovine leg data set. To this end, let us take a look at Figure 5(a), where the measured squared magnitude of the total field (black) and the measured squared magnitude of the incident field (blue) are shown for a given transmitter. On the other hand, Figure 5(b) shows the phase of the total measured (black) and incident (blue) field data for the same transmitter. As can be seen, as opposed to the phase data, the magnitude data of the total and incident fields are quite similar, and therefore, the information content of the imaging experiment is mainly encoded in the phase data that cannot be used for phaseless inversion. This is the main reason behind the difficulty of performing phaseless inversion on this data set. Let us now take a look at the phaseless inversion result using the SL-PGNI algorithm shown in Figure 4(c)-(d). As can be seen this phaseless inversion was relatively unsuccessful, however, if we now take a look

\footnotetext{
${ }^{11}$ This modification was done in an ad hoc manner. In particular, we made the steering parameters smaller for the skinless bovine leg example thereby changing the weight of the regularization operators. For example, consider the steering parameter for the spatial prior case, i.e., the SP-PGNI algorithm. If we make $\gamma_{n}^{2}$ smaller, then the weight of the regularization operator $\mathcal{S}_{n}$ can increase due to having $\gamma_{n}^{2}$ in the denominator of (19).
} 
at the simulated squared magnitude of the total field data at the last iteration of this inversion algorithm, i.e., the red curve in Figure 5(a), we understand that the simulated data are quite similar to the measured magnitude data. Therefore, the phaseless inversion algorithm performed well; however, there was not a great deal of information left to be extracted from the magnitude-only data to improve the reconstruction result. Now, if we look at the simulated phase of the total field data at the last iteration of this phaseless algorithm, i.e., red curve in Figure 5(b), we see that the simulated phase data are different than the phase of the measured total field data. Since these measured phase data were not provided to the SLPGNI inversion algorithm, the algorithm was not able to take advantage of the embedded information content in this dataset to improve its reconstruction.

\section{CONCLUSION}

A novel phaseless GNI algorithm was developed and was augmented with three forms of multiplicative regularization: (i) weighted $L_{2}$ norm total variation regularization, (ii) weighted $L_{2}$ norm total variation regularization combined with prior information regarding the expected complex permittivity values of the objects being imaged, and (iii) weighted $L_{2}$ norm total variation regularization combined with prior information regarding the structural information of the regions of identical permittivity of the objects being imaged. We have referred to these three implementations of the phaseless GNI algorithm as the PGNI, SL-PGNI, and SP-PGNI algorithms, respectively. These algorithms were shown to be able to invert synthetic phaseless data, experimental phaseless data from lossless and lossy targets. The incorporation of prior information was shown to play a strong role with these phaseless GNI algorithms to compensate for the lack of phase of the total field data.

\section{ACKNOWLEDGMENT}

The authors would like to thank the Natural Sciences and Engineering Research Council (NSERC) of Canada, and the Canada Research Chair Program for its financial support. We would also like to thank the Institut Fresnel in France, and the Electromagnetic Imaging Laboratory and Prof. Joe LoVetri at the University of Manitoba for providing the experimental data. Finally, we would like to acknowledge Mr. Nozhan Bayat for his contributions towards the development of the spatial prior regularization scheme.

\section{APPENDIX}

\section{A. Required Derivative Operators}

Herein, we show in more detail how we minimize the phaseless data misfit cost functional $\mathcal{C}(\chi)$ using the GaussNewton inversion framework. In particular, we aim to derive the main (unregularized) update equation given in (9). At the $n^{\text {th }}$ iteration, we update our latest $\chi_{n}$ as $\chi_{n+1}=\chi_{n}+\nu_{n} \Delta \chi_{n}$ by minimizing the regularized form of $\mathcal{C}(\chi)$. This requires the derivative operators of both the phaseless data misfit functional and the regularization terms. Since the regularizations terms for full data (complex) inversion and phaseless inversion are the same, we focus on the required derivative operators for the phaseless data misfit cost functional. The phaseless cost functional $\mathcal{C}(\chi)$ maps the complex vector $\chi$ to a real number and is not analytic with respect to $\chi$ in the complex domain. Therefore, in order to calculate $\Delta \chi_{n}$ within the GNI framework, we use Wirtinger calculus [36], [47]-[49] to define the required derivative operators. This is based on treating $\chi$ and its complex conjugate $\chi^{*}$ as two independent functions (or, vectors in the discrete domain). ${ }^{12}$ The GNI framework calculates the update $\Delta \chi_{n}$ by approximating $\mathcal{C}\left(\chi+\Delta \chi_{n}\right)$ by a quadratic model. The update $\Delta \chi_{n}$ is then found for the minimum of this quadratic model. Within the Wirtinger calculus framework, we consider the cost functional $\mathcal{C}\left(\chi, \chi^{*}\right)=\mathcal{C}(\chi)$, where $\mathcal{C}\left(\chi, \chi^{*}\right)$ is analytic with respect to $\chi$ for a fixed $\chi^{*}$ and also analytic with respect to $\chi *$ for a fixed $\chi$. Minimizing $\mathcal{C}\left(\chi, \chi^{*}\right)$ is equivalent to minimizing $\mathcal{C}(\chi)$. Applying Newton optimization to the phaseless cost functional leads to $\Delta \chi_{n}$ obeying the following relation

$$
\left(\begin{array}{cc}
\frac{\partial^{2} \mathcal{C}}{\partial \chi \partial \chi} & \frac{\partial^{2} \mathcal{C}}{\partial \chi \partial \chi^{*}} \\
\frac{\partial^{2} \mathcal{C}}{\partial \chi^{*} \partial \chi} & \frac{\partial^{2} \mathcal{C}}{\partial \chi^{*} \partial \chi^{*}}
\end{array}\right)\left(\begin{array}{c}
\Delta \chi_{n} \\
\Delta \chi_{n}^{*}
\end{array}\right)=-\left(\begin{array}{c}
\frac{\partial \mathcal{C}}{\partial \chi} \\
\frac{\partial \mathcal{C}}{\partial \chi^{*}}
\end{array}\right)
$$

where $\frac{\partial \mathcal{C}}{\partial \chi}$ and $\frac{\partial \mathcal{C}}{\partial \chi^{*}}$ are the derivative operators with respect to $\chi$ and $\chi^{*}$, respectively at the $n^{\text {th }}$ iteration. These derivative operators, in the discrete domain, act on a complex vector and output a complex number. Similarily, $\frac{\partial^{2} \mathcal{C}}{\partial \chi \partial \chi}$ is a second derivative operator, in this case, with respect to $\chi$ and then $\chi$ again evaluated at the $n^{\text {th }}$ iteration of the algorithm. In the discrete domain, the second order derivative operators act on a complex vector and output a complex vector.

\section{B. First Order Derivative Operators}

In order to derive the required first order derivative operators, we use the following relation

$$
\delta \mathcal{C}=\lim _{\epsilon \rightarrow 0} \frac{\mathcal{C}(\chi+\epsilon \psi)-\mathcal{C}(\chi)}{\epsilon}
$$

where $\epsilon \in \mathbb{R}$ is a scalar, and $\psi$ is an arbitrary function (vector) that is used to modify $\chi$. Since we are treating $\chi$ and $\chi^{*}$ as two independent functions, $\delta C$ will be ${ }^{13}$

$$
\delta \mathcal{C}=\frac{\partial \mathcal{C}}{\partial \chi}(\psi)+\frac{\partial \mathcal{C}}{\partial \chi^{*}}\left(\psi^{*}\right) .
$$

As will be seen later, the two terms on the right hand side of (22) are complex conjugates of each other. Therefore, $\delta \mathcal{C}$ will be a real number. This is expected since $\mathcal{C}(\chi) \in \mathbb{R}$. Also, note that the range of these two first order derivative operators will be complex numbers. In other words, the operation of these derivative operators can be represented by inner products. In our case, the inner product over the ROI is defined as

$$
\langle\varphi, \psi\rangle=\int_{\mathrm{ROI}} \varphi \psi^{*} d s .
$$

\footnotetext{
${ }^{12}$ An alternative approach would be to optimize over the real and imaginary parts of the contrast. This has been shown to be the same as treating $\chi$ and $\chi^{*}$ as two independent functions (vectors), see [36, Appendix D.5].

${ }^{13}$ To understand this better, let us consider a function such as $f(x, y)$, then $\delta f=\frac{\partial f}{\partial x} \delta x+\frac{\partial f}{\partial y} \delta y$. Since we have treated $\chi$ and $\chi^{*}$ as two independent functions, we have a similar form for $\delta \mathcal{C}$ as shown in (22)
} 
Based on this, the right hand side of (22) can be represented by two inner products, from which we can obtain the expression for the first order derivative operators. In (24), we have shown how to write $\delta \mathcal{C}$ based on two inner products. It should be noted that $\frac{\partial E}{\partial \chi}$ in (24) should be evaluated at the current estimate of $\chi$, and 'Re' denotes the real-part operator. For example, when we are at the $n^{\text {th }}$ iteration, this derivative operator should be evaluated at $\chi=\chi_{n}$. In addition, the second order derivative $\frac{\partial^{2} E}{\partial \chi^{2}}$ has been neglected (GNI approximation) in (24). Noting (24) and the definition of the inner product over the ROI given in (23), it is now possible to identify our first order derivative operators. In the discrete domain, the first order derivative operators evaluated at $\chi_{n}$ are

$$
\begin{aligned}
& \left.\frac{\partial \mathcal{C}}{\partial \chi^{*}}\right|_{\chi=\chi_{n}}=2 \zeta \mathbf{J}_{n}^{H}\left[E\left(\chi_{n}\right) \odot\left(\left|E\left(\chi_{n}\right)\right|^{2}-M^{2}\right)\right] \\
& \left.\frac{\partial \mathcal{C}}{\partial \chi}\right|_{\chi=\chi_{n}}=\left(\left.\frac{\partial \mathcal{C}}{\partial \chi^{*}}\right|_{\chi=\chi_{n}}\right)^{*}
\end{aligned}
$$

where $\zeta$ is the normalization coefficient of the phaseless data misfit cost functional, see (8), and $\mathbf{J}_{n}$ denotes the Jacobian (sensitivity) matrix that represents the derivative of the total field data on the measurement domain with respect to the contrast $\chi$ (i.e., $\left.\frac{\partial E}{\partial \chi}\right) .{ }^{14}$

\section{Second Order Derivative Operators}

To calculate the second order derivative operators required to calculate $\Delta \chi_{n}$, we first calculate a second order differential as [36, Appendix D.2]

$$
\delta^{2} \mathcal{C}_{1}=\lim _{\epsilon \rightarrow 0} \frac{\left.\frac{\partial \mathcal{C}}{\partial \chi}\right|_{\chi+\epsilon \psi}(\varphi)-\left.\frac{\partial \mathcal{C}}{\partial \chi}\right|_{\chi}(\varphi)}{\epsilon},
$$

where the vertical lines denote that the derivative operators are evaluated at $\chi+\epsilon \psi$ and $\chi$ respectively. Note that the first order derivative operators are operating on an arbitrary function $\varphi$ (or, vector in the discrete domain). Also, note that the expressions for the first-order derivative operators have been derived in (24), which will now be used to find the second-order derivative operators. Similar to the first order derivative case, some of the intermediate steps are shown in (27). (For simplicity of notation, the derivative operator $\frac{\partial E}{\partial \chi}$ when used without a vertical line indicates that this operator is evaluated at $\chi$ as opposed to at $\chi+\epsilon \psi$.) Using the result in (27), the second order derivative operators can be found by noting that $\delta^{2} C$ can be written as

$$
\begin{aligned}
\delta^{2} \mathcal{C} & =\overbrace{\left[\frac{\partial^{2} \mathcal{C}}{\partial \chi^{*} \partial \chi}(\varphi)\left(\psi^{*}\right)+\frac{\partial^{2} \mathcal{C}}{\partial \chi \partial \chi}(\varphi)(\psi)\right]}^{\delta^{2} \mathcal{C}_{1}} \\
& +\underbrace{\left[\frac{\partial^{2} \mathcal{C}}{\partial \chi^{*} \partial \chi^{*}}\left(\varphi^{*}\right)\left(\psi^{*}\right)+\frac{\partial^{2} \mathcal{C}}{\partial \chi \partial \chi^{*}}\left(\varphi^{*}\right)(\psi)\right]}_{\delta^{2} \mathcal{C}_{2}=\left(\delta^{2} \mathcal{C}_{1}\right)^{*}}=2 \operatorname{Re}\left(\delta^{2} \mathcal{C}_{1}\right) .
\end{aligned}
$$

\footnotetext{
${ }^{14}$ The derivation and expression for the Jacobian matrix can be found in [36, Appendix D.1]. This requires the calculation of the so-called inhomogeneous (or, distorted) Green's function. Also, we remind the reader that the derivative of the total field with respect to the contrast is the same as the derivative of the scattered field with respect to the contrast.
}

Thus, it follows that, in the discrete domain, the second order derivative operators derived from (27) are

$$
\begin{aligned}
& \frac{\partial^{2} \mathcal{C}}{\partial \chi^{*} \partial \chi}(\varphi)\left(\psi^{*}\right)=2 \zeta \mathbf{J}_{n}^{H} \operatorname{diag}\left(2\left|E\left(\chi_{n}\right)\right|^{2}-M^{2}\right) \mathbf{J}_{n}, \\
& \frac{\partial^{2} \mathcal{C}}{\partial \chi \partial \chi}(\varphi)(\psi)=2 \zeta \mathbf{J}_{n}^{T} \operatorname{diag}\left(E^{2}(\chi)\right)^{*} \mathbf{J}_{n} \\
& \frac{\partial^{2} \mathcal{C}}{\partial \chi \partial \chi^{*}}\left(\varphi^{*}\right)(\psi)=\left(\frac{\partial^{2} \mathcal{C}}{\partial \chi^{*} \partial \chi}(\varphi)\left(\psi^{*}\right)\right)^{*}, \\
& \frac{\partial^{2} \mathcal{C}}{\partial \chi^{*} \partial \chi^{*}}\left(\varphi^{*}\right)\left(\psi^{*}\right)=\left(\frac{\partial^{2} \mathcal{C}}{\partial \chi \partial \chi}(\varphi)(\psi)\right)^{*} .
\end{aligned}
$$

In addition to assuming that $\frac{\partial^{2} E}{\partial \chi^{2}}$ is negligible in (27), we make the following extra assumption: $\frac{\partial^{2} \mathcal{C}}{\partial \chi \partial \chi}$ and consequently $\frac{\partial^{2} \mathcal{C}}{\partial \chi^{*} \partial \chi^{*}}$ are disregarded since they are not hermitian (self adjoint) operators. ${ }^{15}$

Based on the above approximation and assumption, (20) at the $n^{\text {th }}$ iteration will simplify to

$$
\left.\frac{\partial^{2} \mathcal{C}}{\partial \chi^{*} \partial \chi}\right|_{\chi=\chi_{n}}\left(\Delta \chi_{n}\right)=-\left.\frac{\partial \mathcal{C}}{\partial \chi^{*}}\right|_{\chi=\chi_{n}} .
$$

Note that the above equation in the continuous domain indicates the equality of two operators. However, in the discrete domain, it represents the equality of two vectors. In particular, using (29) and (25), the discrete form of (30) will become

$$
\begin{gathered}
{\left[2 \zeta \mathbf{J}_{n}^{H} \operatorname{diag}\left(2\left|E\left(\chi_{n}\right)\right|^{2}-M^{2}\right) \mathbf{J}_{n}\right] \Delta \chi_{n}=} \\
\quad-2 \zeta \mathbf{J}_{n}^{H}\left[E\left(\chi_{n}\right) \odot\left(\left|E\left(\chi_{n}\right)\right|^{2}-M^{2}\right)\right] .
\end{gathered}
$$

This is the main update equation (in un-regularized form) which was shown in (9).

\footnotetext{
${ }^{15}$ Note that this extra assumption was not needed in the development of the complex GNI algorithm [36, Equation (D.33)]. In fact, in the complex GNI algorithm, the assumption of a negligible $\frac{\partial^{2} E}{\partial \chi^{2}}$ is sufficient to have $\frac{\partial^{2} \mathcal{C}}{\partial \chi \partial \chi}$ and $\frac{\partial^{2} \mathcal{C}}{\partial \chi^{*} \partial \chi^{*}}$ as zero.
} 


$$
\begin{aligned}
\delta \mathcal{C} & =\lim _{\epsilon \rightarrow 0} \frac{\zeta}{\epsilon}\left(\left\|M^{2}-|E(\chi+\epsilon \psi)|^{2}\right\|^{2}-\left\|M^{2}-|E(\chi)|^{2}\right\|^{2}\right) \\
& =\lim _{\epsilon \rightarrow 0} \frac{\zeta}{\epsilon}\left(\left\|M^{2}-\left|E(\chi)+\epsilon \frac{\partial E}{\partial \chi} \psi\right|^{2}\right\|^{2}-\left\|M^{2}-|E(\chi)|^{2}\right\|^{2}\right) \\
& =\lim _{\epsilon \rightarrow 0} \frac{\zeta}{\epsilon}\left(\left\|M^{2}-\left(|E(\chi)|^{2}+\left|\epsilon \frac{\partial E}{\partial \chi} \psi\right|^{2}+2 \epsilon \operatorname{Re}\left\{E(\chi)^{*} \frac{\partial E}{\partial \chi} \psi\right\}\right)\right\|^{2}-\left\|M^{2}-|E(\chi)|^{2}\right\|^{2}\right) \\
& =\lim _{\epsilon \rightarrow 0} \frac{\zeta}{\epsilon}\left(4 \epsilon^{2}|| \operatorname{Re}\left\{E(\chi)^{*} \frac{\partial E}{\partial \chi} \psi\right\} \|^{2}+2 \operatorname{Re}\left\langle M^{2}-|E(\chi)|^{2},-2 \zeta \epsilon \operatorname{Re}\left\{E(\chi)^{*} \frac{\partial E}{\partial \chi} \psi\right\}\right\rangle\right) \\
& =-2 \zeta \operatorname{Re}\left\langle M^{2}-|E(\chi)|^{2}, 2 \operatorname{Re}\left\{E(\chi)^{*} \frac{\partial E}{\partial \chi} \psi\right\}\right\rangle \\
& =-2 \zeta\left\langle M^{2}-|E(\chi)|^{2}, E(\chi)^{*} \frac{\partial E}{\partial \chi} \psi+E(\chi)\left(\frac{\partial E}{\partial \chi} \psi\right)^{*}\right\rangle \\
& =-2 \zeta\left\langle M^{2}-|E(\chi)|^{2}, E(\chi)^{*} \frac{\partial E}{\partial \chi} \psi\right\rangle-2 \zeta\left\langle M^{2}-|E(\chi)|^{2}, E(\chi)\left(\frac{\partial E}{\partial \chi} \psi\right)^{*}\right\rangle \\
& =\underbrace{\left\langle 2 \zeta\left(\frac{\partial E}{\partial \chi}\right)^{a}\left(E(\chi)\left(|E(\chi)|^{2}-M^{2}\right)\right), \psi\right\rangle}_{\frac{\partial \mathcal{C}}{\partial \chi^{*}}\left(\psi^{*}\right)}+\left\langle 2 \zeta\left(\left(\frac{\partial E}{\partial \chi}\right)^{a}\left(E(\chi)\left(|E(\chi)|^{2}-M^{2}\right)\right)\right)^{*}, \psi^{*}\right\rangle
\end{aligned}
$$

$$
\begin{aligned}
& \delta^{2} \mathcal{C}_{1}=\lim _{\epsilon \rightarrow 0} \frac{1}{\epsilon}\left[\left\langle\left. 2 \zeta \frac{\partial E}{\partial \chi}\right|_{\chi+\epsilon \psi}(\varphi),\left[E(\chi+\epsilon \psi)\left(|E(\chi+\epsilon \psi)|^{2}-M^{2}\right)\right]\right\rangle-\left\langle 2 \zeta \frac{\partial E}{\partial \chi}(\varphi),\left[E(\chi)\left(|E(\chi)|^{2}-M^{2}\right)\right]\right\rangle\right] \\
& =\lim _{\epsilon \rightarrow 0} \frac{1}{\epsilon}\left[2 \zeta\left\langle\left(\frac{\partial E}{\partial \chi}+\epsilon \frac{\partial^{2} E}{\partial \chi^{2}}(\psi)\right)(\varphi),\left(E(\chi)+\epsilon \frac{\partial E}{\partial \chi}(\psi)\right)\left(\left|E(\chi)+\epsilon \frac{\partial E}{\partial \chi}(\psi)\right|^{2}-M^{2}\right)\right\rangle \ldots\right. \\
& \left.-2 \zeta\left\langle\frac{\partial E}{\partial \chi}(\varphi), E(\chi)\left(|E(\chi)|^{2}-M^{2}\right)\right\rangle\right] \\
& =\lim _{\epsilon \rightarrow 0} \frac{1}{\epsilon}\left[2 \zeta\left\langle\frac{\partial E}{\partial \chi}(\varphi),\left(E(\chi)+\epsilon \frac{\partial E}{\partial \chi}(\psi)\right)\left(|E(\chi)|^{2}+\epsilon^{2}\left|\frac{\partial E}{\partial \chi}(\psi)\right|^{2}+2 \epsilon \operatorname{Re}\left(E(\chi)^{*} \frac{\partial E}{\partial \chi}(\psi)\right)-M^{2}\right)\right\rangle \ldots\right. \\
& \left.-2 \zeta\left\langle\frac{\partial E}{\partial \chi}(\varphi), E(\chi)\left(|E(\chi)|^{2}-M^{2}\right)\right\rangle\right] \\
& =\lim _{\epsilon \rightarrow 0} \frac{1}{\epsilon}\left[2 \zeta\left\langle\frac{\partial E}{\partial \chi}(\varphi),\left(E(\chi) 2 \epsilon \operatorname{Re}\left(E(\chi)^{*} \frac{\partial E}{\partial \chi}(\psi)\right)\right)\right\rangle+2 \zeta\left\langle\frac{\partial E}{\partial \chi}(\varphi), \epsilon \frac{\partial E}{\partial \chi}(\psi)\left(|E(\chi)|^{2}-M^{2}\right)\right\rangle\right] \\
& =2 \zeta\left\langle\frac{\partial E}{\partial \chi}(\varphi), E(\chi) E(\chi)^{*} \frac{\partial E}{\partial \chi}(\psi)\right\rangle+2 \zeta\left\langle\frac{\partial E}{\partial \chi}(\varphi), E(\chi) E(\chi)\left[\frac{\partial E}{\partial \chi}(\psi)\right]^{*}\right\rangle+2 \zeta\left\langle\frac{\partial E}{\partial \chi}(\varphi), \frac{\partial E}{\partial \chi}(\psi)\left(|E(\chi)|^{2}-M^{2}\right)\right\rangle \\
& =\underbrace{\left\langle 2 \zeta\left(\frac{\partial E}{\partial \chi}\right)^{a}\left(2|E(\chi)|^{2}-M^{2}\right) \frac{\partial E}{\partial \chi}(\varphi), \psi\right\rangle}_{\frac{\partial^{2} \mathcal{C}}{\partial \chi^{*} \partial \chi}(\varphi)\left(\psi^{*}\right)}+\underbrace{\left\langle 2 \zeta\left(\left(\frac{\partial E}{\partial \chi}\right)^{a} E^{2}(\chi)\left(\frac{\partial E}{\partial \chi}(\varphi)\right)^{*}\right)^{*}, \psi^{*}\right\rangle}_{\frac{\partial^{2} \mathcal{C}}{\partial \chi \partial \chi}(\varphi)(\psi)}
\end{aligned}
$$

\section{REFERENCES}

[1] S. P. Poplack, T. D. Tosteson, W. A. Wells, B. W. Pogue, P. M. Meaney, A. Hartov, C. A. Kogel, S. K. Soho, J. J. Gibson, and K. D. Paulsen, "Electromagnetic breast imaging: results of a pilot study in women with abnormal mammograms," Radiology, vol. 243, no. 2, pp. 350-359, 2007.

[2] E. C. Fear, S. C. Hagness, P. M. Meaney, M. Okoniewski, and M. A. Stuchly, "Enhancing breast tumor detection with near-field imaging," IEEE Microwave Mag., vol. 3, no. 1, pp. 48-56, Mar 2002.
[3] W. H. Weedon, W. C. Chew, and P. E. Mayes, "A step-frequency radar imaging system for microwave nondestructive evaluation," Progress In Electromagnetics Research, vol. 28, pp. 121-146, 2000.

[4] N. Firoozy, T. Neusitzer, D. S. Desmond, T. Tiede, M. J. L. Lemes, J. Landy, P. Mojabi, S. Rysgaard, G. Stern, and D. G. Barber, "An electromagnetic detection case study on crude oil injection in a young sea ice environment," IEEE Transactions on Geoscience and Remote Sensing, vol. 55, no. 8, pp. 4465-4475, Aug 2017. 
[5] M. Ostadrahimi, P. Mojabi, C. Gilmore, A. Zakaria, S. Noghanian, S. Pistorius, and J. LoVetri, "Analysis of incident field modeling and incident/scattered field calibration techniques in microwave tomography," IEEE Antennas and Wireless Propagation Letters, vol. 10, pp. 900-903, 2011.

[6] A. Abubakar, P. M. van den Berg, and J. J. Mallorqui, "Imaging of biomedical data using a multiplicative regularized contrast source inversion method," IEEE Trans. Microwave Theory Tech., vol. 50, no. 7, pp. 1761-1777, July 2002.

[7] A. Abubakar, T. Habashy, V. Druskin, L. Knizhnerman, and D. Alumbaugh, "2.5D forward and inverse modeling for interpreting lowfrequency electromagnetic measurements," Geophysics, vol. 73, no. 4, pp. F165-F177, 2008

[8] P. Mojabi and J. LoVetri, "Microwave biomedical imaging using the multiplicative regularized Gauss-Newton inversion," IEEE Antennas and Wireless Propagation Letters, vol. 8, pp. 645-648, 2009.

[9] A. Abubakar and P. M. van den Berg, "Iterative forward and inverse algorithms based on domain integral equations for three-dimensional electric and magnetic objects," J. Comput. Phys., vol. 195, pp. 236-262, 2004.

[10] P. Mojabi and J. LoVetri, "Overview and classification of some regularization techniques for the Gauss-Newton inversion method applied to inverse scattering problems," IEEE Transactions on Antennas and Propagation, vol. 57, no. 9, pp. 2658-2665, Sept 2009.

[11] A. Abubakar and P. M. van den Berg, "The contrast source inversion method for location and shape reconstructions," Inverse Problems, vol. 18 , no. 2 , pp. 495-510, Mar 2002.

[12] P. Mojabi, J. LoVetri, and L. Shafai, "A multiplicative regularized Gauss-Newton inversion for shape and location reconstruction," IEEE Transactions on Antennas and Propagation, vol. 59, no. 12, pp. 47904802, 2011

[13] S. F. Razavi and Y. Rahmat-Samii, "Resilience to probe-positioning errors in planar phaseless near-field measurements," IEEE Transactions on Antennas and Propagation, vol. 58, no. 8, pp. 2632-2640, 2010.

[14] L. Li, H. Zheng, and F. Li, "Two-dimensional contrast source inversion method with phaseless data: TM case," IEEE Transactions on Geoscience and Remote Sensing, vol. 47, no. 6, pp. 1719-1736, 2009.

[15] Z. Hu, L. Lianlin, and L. Fang, "A multi-frequency MRCSI algorithm with phaseless data," Inverse Problems, vol. 25, no. 6, p. 065006, 2009.

[16] M. H. Maleki, A. J. Devaney, and A. Schatzberg, "Tomographic reconstruction from optical scattered intensities," JOSA A, vol. 9, no. 8, pp. 1356-1363, 1992.

[17] M. H. Maleki and A. J. Devaney, "Phase-retrieval and intensity-only reconstruction algorithms for optical diffraction tomography," JOSA A, vol. 10 , no. 5, pp. 1086-1092, 1993

[18] T. Takenaka, D. J. Wall, H. Harada, and M. Tanaka, "Reconstruction algorithm of the refractive index of a cylindrical object from the intensity measurements of the total field," Microwave and optical technology letters, vol. 14, no. 3, pp. 182-188, 1997.

[19] G. Gbur and E. Wolf, "Hybrid diffraction tomography without phase information," JOSA A, vol. 19, no. 11, pp. 2194-2202, 2002.

[20] S. Caorsi, A. Massa, M. Pastorino, and A. Randazzo, "Electromagnetic detection of dielectric scatterers using phaseless synthetic and real data and the memetic algorithm," IEEE transactions on geoscience and remote sensing, vol. 41, no. 12, pp. 2745-2753, 2003.

[21] L. Crocco, M. DUrso, and T. Isernia, "Inverse scattering from phaseless measurements of the total field on a closed curve," JOSA A, vol. 21 , no. 4, pp. 622-631, 2004.

[22] G. Franceschini, M. Donelli, R. Azaro, and A. Massa, "Inversion of phaseless total field data using a two-step strategy based on the iterative multiscaling approach," IEEE Transactions on Geoscience and Remote Sensing, vol. 44, no. 12, pp. 3527-3539, 2006.

[23] A. Litman and K. Belkebir, "Two-dimensional inverse profiling problem using phaseless data," JOSA A, vol. 23, no. 11, pp. 2737-2746, 2006.

[24] O. M. Bucci, L. Crocco, M. D’Urso, and T. Isernia, "Inverse scattering from phaseless measurements of the total field on open lines," JOSA A, vol. 23 , no. 10, pp. 2566-2577, 2006.

[25] M. d'Urso, K. Belkebir, L. Crocco, T. Isernia, and A. Litman, "Phaseless imaging with experimental data: facts and challenges," JOSA A, vol. 25, no. 1, pp. 271-281, 2008.

[26] L. Li, W. Zhang, and F. Li, "Tomographic reconstruction using the distorted Rytov iterative method with phaseless data," IEEE Geoscience and Remote Sensing Letters, vol. 5, no. 3, pp. 479-483, 2008.

[27] W. Zhang, L. Li, and F. Li, "Multifrequency imaging from intensity-only data using the phaseless data distorted Rytov iterative method," IEEE Transactions on Antennas and Propagation, vol. 57, no. 1, pp. 290-295, 2009.
[28] R. G. Yaccarino and Y. Rahmat-Samii, "Phaseless bi-polar planar nearfield measurements and diagnostics of array antennas," IEEE Transactions on Antennas and Propagation, vol. 47, no. 3, pp. 574-583, March 1999.

[29] T. Brown, I. Jeffrey, and P. Mojabi, "Multiplicatively regularized source reconstruction method for phaseless planar near-field antenna measurements," IEEE Transactions on Antennas and Propagation, vol. 65, no. 4, pp. 2020-2031, 2017.

[30] T. Rubæk, P. M. Meaney, P. Meincke, and K. D. Paulsen, "Nonlinear microwave imaging for breast-cancer screening using Gauss-Newton's method and the CGLS inversion algorithm," IEEE Trans. Antennas Propag., vol. 55, no. 8, pp. 2320-2331, Aug 2007.

[31] C. Gilmore, P. Mojabi, A. Zakaria, M. Ostadrahimi, C. Kaye, S. Noghanian, L. Shafai, S. Pistorius, and J. LoVetri, "A wideband microwave tomography system with a novel frequency selection procedure," IEEE Transactions on Biomedical Engineering, vol. 57, no. 4, pp. 894-904, April 2010.

[32] S. Costanzo and G. Di Massa, "Wideband phase retrieval technique from amplitude-only near-field data." Radioengineering, vol. 17, no. 4, 2008.

[33] S. Costanzo, G. Di Massa, M. Pastorino, and A. Randazzo, "Hybrid microwave approach for phaseless imaging of dielectric targets," IEEE Geoscience and Remote Sensing Letters, vol. 12, no. 4, pp. 851-854, April 2015.

[34] T. M. Habashy and A. Abubakar, "A general framework for constraint minimization for the inversion of electromagnetic measurements," Progress in electromagnetics Research, vol. 46, pp. 265-312, 2004.

[35] M. Ostadrahimi, P. Mojabi, A. Zakaria, J. LoVetri, and L. Shafai, "Enhancement of Gauss-Newton inversion method for biological tissue imaging," IEEE Transactions on Microwave Theory and Techniques, vol. 61 , no. 9, pp. 3424-3434, 2013.

[36] P. Mojabi, "Investigation and development of algorithms and techniques for microwave tomography," Ph.D. dissertation, University of Manitoba, Winnipeg, Manitoba, Canada (Available Online), 2010.

[37] N. Bayat and P. Mojabi, "Incorporating spatial priors in microwave imaging via multiplicative regularization," IEEE Transactions on Antennas and Propagation, 2019.

[38] P. Mojabi and N. Bayat, "A multiplicative regularizer to incorporate prior spatial data in microwave imaging reconstruction," in 13th European Conference on Antennas and Propagation, April 2019, pp. 1-5.

[39] A. H. Golnabi, P. M. Meaney, S. D. Geimer, and K. D. Paulsen, "3d microwave tomography using the soft prior regularization technique: Evaluation in anatomically realistic mri-derived numerical breast phantoms," IEEE Transactions on Biomedical Engineering, vol. 66, no. 9, pp. 2566-2575, Sep. 2019

[40] L. M. Neira, B. D. Van Veen, and S. C. Hagness, "High-resolution microwave breast imaging using a 3-d inverse scattering algorithm with a variable-strength spatial prior constraint," IEEE Transactions on Antennas and Propagation, vol. 65, no. 11, pp. 6002-6014, Nov 2017.

[41] A. H. Golnabi, P. M. Meaney, and K. D. Paulsen, "Tomographic microwave imaging with incorporated prior spatial information," IEEE Transactions on Microwave Theory and Techniques, vol. 61, no. 5, pp. 2129-2136, May 2013

[42] C. Gilmore, A. Zakaria, P. Mojabi, M. Ostadrahimi, S. Pistorius, and J. LoVetri, "The University of Manitoba microwave imaging repository: A two-dimensional microwave scattering database for testing inversion and calibration algorithms [Measurements Corner]," IEEE Antennas and Propagation Magazine, vol. 53, no. 5, pp. 126-133, Oct 2011.

[43] T. Brown, C. Narendra, C. Niu, and P. Mojabi, "On the use of electromagnetic inversion for near-field antenna measurements: A review," in 2018 IEEE Conference on Antenna Measurements Applications (CAMA), Sep. 2018, pp. $1-4$.

[44] C. Narendra, I. Jeffrey, and P. Mojabi, "Using the source reconstruction method to model incident fields in microwave tomography," IEEE Antennas and Wireless Propagation Letters, vol. 16, pp. 46-49, 2016.

[45] P. C. Hansen, "Analysis of discrete ill-posed problems by means of the L-curve," SIAM Review, vol. 34, no. 4, pp. 561-580, Dec 1992.

[46] J.-M. Geffrin, P. Sabouroux, and C. Eyraud, "Free space experimental scattering database continuation: experimental set-up and measurement precision," inverse Problems, vol. 21, no. 6, p. S117, 2005.

[47] A. Van Den Bos, "Complex gradient and hessian," IEE ProceedingsVision, Image and Signal Processing, vol. 141, no. 6, pp. 380-382, 1994.

[48] P. Mojabi and J. LoVetri, "Use of Wirtinger calculus in Gauss-Newton inversion of microwave tomography data," in 2014 XXXIth URSI General Assembly and Scientific Symposium (URSI GASS), Aug 2014, pp. $1-4$.

[49] J. De Zaeytijd, A. Franchois, C. Eyraud, and J. Geffrin, "Full-wave three-dimensional microwave imaging with a regularized Gauss-Newton 
method - Theory and experiment," IEEE Transactions on Antennas and Propagation, vol. 55, no. 11, pp. 3279-3292, Nov 2007. 